(1)

CrossMark

\title{
Towards precision medicine: CCL2, another brick in the wall?
}

\author{
Andriani Charpidou , Elias Kotteas ${ }^{1}$ and Mina Gaga ${ }^{2}$
}

Affiliations: ${ }^{1}$ Oncology Unit, 3rd Internal Medicine Dept, Medical School, National Kapodistrian University of Athens, Athens Chest Hospital Sotiria, Athens, Greece. ${ }^{2} 7$ th Respiratory Medicine Dept, Athens Chest Hospital Sotiria, Athens, Greece.

Correspondence: Mina Gaga, 7th Respiratory Medicine Dept, Athens Chest Hospital Sotiria, Athens, Greece. E-mail: minagagadyahoo.com

@ERSpublications

Research for predictive biomarkers that are crucial to decisions for targeted treatments is very important; the data should be validated in large studies http://ow.ly/mzis30neEA6

Cite this article as: Charpidou A, Kotteas E, Gaga M. Towards precision medicine: CCL2, another brick in the wall? Eur Respir J 2019; 53: 1802327 [https://doi.org/10.1183/13993003.02327-2018].

The introduction of targeted treatment in lung cancer has substantially changed the outcome of disease and the lives of lung cancer patients. Having biomarkers that can identify the right candidates and that predict the response to a specific treatment is very important and currently, research focuses on the identification of reliable molecular markers. In this issue of the European Respiratory Journal, Lu et al. [1] provide insight into the mechanisms of action of a new medication, anlotinib, and propose a new, predictive biomarker, the C-C motif ligand 2 (CCL2).

Anlotinib is a multi-target tyrosine kinase inhibitor; it acts on vascular endothelial growth factor receptor (VEGFR), platelet-derived growth factor receptors, fibroblast growth factor receptor and c-kit. A previous study from the same group, showed that anlotinib therapy was beneficial in prolonging overall survival in heavily pre-treated nonsmall cell lung cancer (NSCLC) patients [2]. In the current study by Lu et al. [1], the authors retrospectively screened for the presence of biomarkers of anlotinib response in a subgroup of patients from the original cohort. The study showed that anlotinib therapy was beneficial in prolonging overall survival in NSCLC patients harbouring positive driver gene mutations, especially patients harbouring the epidermal growth factor receptor (EGFR) T790M mutation. Moreover, the researchers found that benefits in progression-free and overall survival in refractory advanced NSCLC were associated with anlotinib-induced serum CCL2 level decreases. So, the study indicates that patients exhibiting specific mutations respond to targeted treatment, in line with the current treatment practice and, importantly, the authors report a novel anti-angiogenetic mechanism of anlotinib via inhibiting CCL2. They also suggest that changes in serum CCL2 levels may be used to monitor and predict clinical outcomes of anlotinib administration in refractory advanced NSCLC patients $[1,2]$.

Having predictive markers is pivotal when deciding on medications that can affect the patients' survival and that are expensive and potentially toxic. Over the past decade, our understanding about cancer as well as the treatment choices for its management have grown exponentially. Research into lung cancer in particular, has become a pioneer into precision medicine efforts. Since 2004, when the first specific activating mutations within the tyrosine kinase domain of the EGFR gene were identified and shown to correlate to a dramatic response to EGFR-TKI treatment, huge progress has been made [3]. Today, guidelines and recommendations suggest evaluation of EGFR, ALK, and ROS1 for all patients with metastatic nonsquamous lung cancer, irrespective of their clinical characteristics, and, targeted therapy is 
preferred over chemotherapy when these mutations are detected [4]. Not only EGFR, ALK and ROS1 status but also a broader panel of genes can be tested, including BRAF, RET, ERBB2 and MET, and this testing will be more accessible when next-generation sequencing is available. This is important as one or more targeted therapies are available for patients who exhibit these gene alterations. And, more recently, programmed death-ligand 1 (PD-L1) expression has been added as a molecular marker, since new treatments using anti-programmed cell death-1 antibodies have been approved as first-line monotherapy in the subpopulation of patients with a high tumour proportion score, and PD-L1 positivity in $>50 \%$ of tumour cells [5].

Angiogenesis is a survival mechanism for tumours. With the deeper understanding of the role of angiogenesis in lung cancer progression and metastasis, drugs that have an inhibitory action on the vascular endothelium have been incorporated into lung cancer treatment. An example of such preparations is the anti-VEGF monoclonal antibody, bevacizumab [6]. Other anti-angiogenetic agents, such as ramucirumab [7] and nintedanib [8], are being tested as candidates to be added into the NSCLC treatment armamentarium. However, the initial enthusiasm regarding the potential of these drugs has faded rapidly both because of their modest increase in survival rates, but, mainly, due to the lack of predictive biomarkers. Although of great clinical importance, the implementation of molecular biomarkers that can predict the responses to inhibitors of angiogenesis in practice still remains something of a treasure hunt; such biomarkers are very difficult to characterise and validate. Previous randomised controlled trials have evaluated the importance of VEGF genetic variants and of soluble adhesion molecules, such as intercellular adhesion molecule (ICAM)-1, levels in plasma, through polymerase chain reaction assays, before and after chemotherapy/VEGF antibody combination administration [9-11]. Higher baseline soluble VEGFR and/or ICAM-1 levels were associated with poorer outcomes. However, it has not been proven that these molecules can be used as independent predictive biomarkers in lung cancer, and further evaluation is required [9-12]. The interesting aspect of the study by Lu et al. [1] is that they evaluated CCL2 as a marker for monitoring and predicting the clinical outcomes of anlotinib therapy. Moreover, as previously mentioned, the researchers used serum and not tissue and this could be an important asset. The increased need for molecular signatures makes the availability of tissue pivotal; nevertheless, it is often critical to avoid invasive techniques in patients who might have a relatively poor performance status. Unfortunately, the authors only managed to use samples from 14 of the 296 patients randomised on the anlotinib arm of the original study; thus the results, although interesting, cannot be considered conclusive. If these results are confirmed by larger trials, then an unmet goal of precision administration of anti-angiogenic factors could be fulfilled.

Conflict of interest: A. Charpidou has nothing to disclose. E. Kotteas has nothing to disclose. M. Gaga reports receiving the following, outside the submitted work: a grant and honoraria from AstraZeneca and Novartis; honoraria from GSK, BMS, MSD and Pharmaten; and a grant from Chiesi.

\section{References}

$1 \mathrm{Lu} \mathrm{J}$, Zhong $\mathrm{H}$, Chu $\mathrm{T}$, et al. Role of anlotinib-induced CCL2 decrease in anti-angiogenesis and response prediction for nonsmall cell lung cancer therapy. Eur Respir J 2019; 53: 1801562.

2 Han B, Li K, Wang Q, et al. Effect of anlotinib as a third-line or further treatment on overall survival of patients with advanced non-small cell lung cancer. JAMA Oncol 2018; 4: 1569-1575.

3 Lynch TJ, Bell DW, Sordella R, et al. Activating mutations in the epidermal growth factor receptor underlying responsiveness of non-small-cell lung cancer to gefitinib. N Engl J Med 2004; 350: 2129-2139.

4 Lindeman NI, Cagle PT, Aisner DL, et al. Updated molecular testing guideline for the selection of lung cancer patients for treatment with targeted tyrosine kinase inhibitors. J Thorac Oncol 2018; 13: 323-358.

5 Reck M, Rodríguez-Abreu D, Robinson AG, et al. Pembrolizumab versus chemotherapy for PD-L1-positive nonsmall-cell lung cancer. N Engl J M 2016; 375: 1823-1833.

6 Sandler A, Gray R, Perry MC, et al. Paclitaxel-carboplatin alone or with bevacizumab for non-small-cell lung cancer. N Engl J Med 2006; 335: 2542-2550.

7 Garon EB, Ciuleanu TE, Arrita $\mathrm{O}$, et al. Ramucirumab plus docetaxel versus placebo plus docetaxel for second-line treatment of stage IV non-small-cell lung cancer after disease progression on platinum-based therapy (REVEL): a multicentre, double-blind, randomised phase 3 trial. Lancet 2014; 384: 665-673.

8 Reck M, Kaiser R, Mellemgaard A, et al. Docetaxel plus nintedanib versus docetaxel plus placebo in patients with previously treated non-small-cell lung cancer (LUME-Lung 1): a phase 3, double-blind, randomised controlled trial. Lancet Oncol 2014; 15: 143-155.

9 Mok T, Gorbunova V, Juhasz E, et al. Combination of bevacizumab and carboplatin-based chemotherapy for advanced nonsquamous non-small cell lung cancer. Biomarker results of the phase II randomized ABIGAIL study (BO21015). J Thorac Oncol 2014; 9: 848-855.

10 Dowlati A, Gray R, Sandler AB, et al. Cell adhesion molecules, vascular endothelial growth factor, and basic fibroblast growth factor in patients with non-small cell lung cancer treated with chemotherapy with or without bevaciumab - an Eastern Cooperative Group Study. Clin Cancer Res 2008; 14: 1407-1412.

11 Pallaud C, Reck M, Juhasz E, et al. Clinical genotyping and efficacy outcomes: exploratory biomarker data from the phase II ABIGAIL study of first-line bevacizumab plus chemotherapy in non-squamous non-small-cell lung cancer. Lung Cancer 2014; 86: 67-72.

12 Crino L, Metro G. Therapeutic options targeting angiogenesis in nonsmall cell lung cancer. Eur Respir Rev 2014; 23: 79-91. 